\title{
COMMUNICATIONS
}

\section{USING REDUCED INTERFERENCE DISTRIBUTION TO ANALYZE ABNORMAL CARDIAC SIGNAL}

\begin{abstract}
Allam Mousa * Rashid Saleem ${ }^{* *}$
Due to the non-stationary, multicomponent nature of biomedical signals, the use of time-frequency analysis can be inevitable for these signals. The choice and selection of the proper Time-Frequency Distribution (TFD) that can reveal the exact multicomponent structure of biological signals is vital in many applications, including the diagnosis of medical abnormalities. In this paper, the instantaneous frequency techniques using two distribution functions are applied for analysis of biological signals. These distributions are the Wigner-Ville Distribution and the Bessel Distribution. The simulation performed on normaland abnormal cardiac signals show that the Bessel Distribution can clearly detect the QRS complexes. However, Wigner-Ville Distribution was able to detect the QRS complexes in the normal signa, but fails to detect these complexes in the abnormal cardiac signal.
\end{abstract}

K e y w or d s: time frequency distribution, cardiac signal, Wigner-Ville distribution, ECG, TFD

\section{INTRODUCTION}

Biological signals typically arise from nonlinear systems, are nonstationary, and have multicomponents. Investigation of such biological signals can assist the surgeon in deciding on surgical intervention [1]. Most of the time, biological signals accompanied by noise, that may consist of artifacts or interferences generated by human body. These facts have motivated the use of frequency domain techniques, such as Fourier transform (FT), for the analysis [2]. However, as the ECG and all other biological signals belong to the family of multicomponent nonstationary signals, accurate time-varying spectral estimates can be extremely difficult to obtain [3]. However, a proper time-frequency distribution (TFD) can tackle this problem and reveal the multicomponent nature of such signals.

Conventionally, electrocardiogram (ECG) signal is analyzed in the time-domain by experienced physicians. However, pathological conditions may not always be obvious in the time-domain signal. This fact has motivated the use of frequency domain techniques, such as Fourier transform (FT), for analysis. However, as the ECG and all other biological signals belong to the family of multi component non stationary signals, accurate time-varying spectral estimates can be extremely difficult to obtain. However, a proper time-frequency distribution (TFD) can deal with this problem and reveal the multi component nature of such signals. The time-frequency presentation provides information where the time-domain and frequency-domain may fail to produce. In particular, it uses to detect the QRS complex signals. Hence, a proper time-frequency distribution can tackle this problem and reveal the multi component nature of such signals.

Time-frequency analysis techniques, in different ways, are used to reveal the multicomponent nature of nonstationary biomedical signals. The instantaneous frequency (IF) and instantaneous bandwidth is an important concept in time-frequency analysis, especially when analyzing multicomponent signals. The concept of the instantaneous frequency can be found in [4-7]. Every TimeFrequency Distribution (TFD) has a ridge or concentration of energy in the time-frequency plane around the instantaneous frequency (IF) of each component. As such, it can be used to estimate the frequency variation of the ECG (or any biomedical) signal over time. There are many TFDs in active use, the most significant class of TFDs is known as the quadratic class or Cohen's class [4]. However, quadratic time-frequency distributions suffer from the presence of cross-terms when used to analyze multicomponent signals [4], [5], [7]. Cross-terms are fictitious concentrations of energy, resulting from the quadratic nature of Cohen's class, which can obscure the real features of interest in the signal. On the other hand, joint time-frequency resolution is another significant character that is different for different TFDs. In some applications (eg, biomedical signal analysis), we may be confronted by multiple components with narrow separation in time, frequency or both; in such a case, many TFDs fail to reveal the true structure, as many components will overlap due to bad resolution. Considerable efforts have been made to define TFDs, which reduce the effect of cross-terms while improving the time-frequency resolution [4], [7]. However, there is always a compromise be-

\footnotetext{
* Electrical Engineering Department, An Najah University, allam@najah.edu, ${ }^{* *}$ School of Electrical and Electronic Engineering, University of Manchester, UK
} 
tween these two requirements. TFD's have different performances in this respect and the choice of the proper TFD is application-dependent.

The Wigner-Ville distribution (WVD) was first defined by E. Wigner in the context of quantum mechanics, and later independently introduced by J. Ville for signal processing and spectral analysis. Though WignerVille gives high resolution in time-frequency domain, it is not used widely for practical application due to the interaction between different signal components, the so-called cross term. Reduced Interference Distributions (RID), such as the Choi-William, Binomial, and Bessel, suppress cross-term formation providing more interpretable results for multicomponent signals [8], [9]. In this paper, we suggest a Bessel function distribution of RID type, based on Bessel distribution. By controlling a parameter of the Bessel kernel in Bessel distribution, both signals can be correctly processed.

\section{CARDIAC SIGNALS}

The ECG signal has a well-defined P, QRS, T signature that represents with each heart beat. The $\mathrm{P}$-wave arises from the depolarization of the atrium. The QRS complex arises from depolarization of the ventricles and $\mathrm{T}$-wave arises from repolarization of the ventricle muscles. The duration, shape and amplitude of these waves are considered as major features in time domain analysis. Sometimes, the time morphologies of these waves are similar. An arrhythmia is an abnormality in the heart's rhythm, or heart beat pattern. The heart beat can be too slow, too fast, have extra beats, or otherwise beat irregularly. Supraventricular arrhythmia occurs in the upper areas of the heart and is less serious than ventricular arrhythmia. It has irregular shapes of QRS complexes.

In this study, two types of biological signals are considered; Normal ECG signal and Abnormal ECG signal for a patient with heart fail [8]. Utilization of time-frequency analysis as a preprocessing stage is extremely important for both signals. IF estimation of a ECG signal will reveal all of its structural components and clearly provide the frequency components of the ECG signal (P-QRS-T signature) as well as their time of occurrence. This will help in the removal of these artifacts from the signal. Due to its high time-frequency resolution and cross-terms control, the RID's expected to be efficient in analyzing more delicate biological signals such as the ECG signal.

\section{TIME FREQUENCY ANALYSIS TECHNIQUES}

The basic objective of time-frequency analysis is to devise a function that will describe the energy density of a signal simultaneously in time and frequency and that can be used and manipulated in the same manner as any density. If we had such a distribution then one could ask for the fraction of the energy in a certain frequency and time range, one could calculate the density of frequency at a particular instant of time, and may calculate the global and local moments of the distribution such as the mean conditional frequency and its local spread and so on.

Time-frequency analysis allows a joint time-frequency resolution. However, when used for analyzing multicomponent signals, this approach is susceptible to cross-terms arising in the middle between the time-frequency (energy) components. Therefore, a TFD that provides a good reduction of the cross terms is needed to make sure that the off-diagonal elements of the TFD matrix of the sources are negligible and that a diagonal structure can be maintained. The continuous time frequency distribution of the analytic signal $\mathrm{z}(\mathrm{t})$ associated with the original real signal $\mathrm{s}(\mathrm{t})$ can be expressed as follows [5], [11]

$$
\rho(t, f)=\underset{\tau \rightarrow f}{\mathbb{F}}\left[G(t, \tau) \underset{(t)}{*} K_{z}(t, \tau)\right]
$$

where $K_{z}(t, \tau)=z(t+\tau / 2) z^{*}(t-\tau / 2)$ is instantaneous autocorrelation product, $\mathbb{F}$ is the Fourier transform, $G(t, \tau)$ is the time-lag kernel, and * denotes time $(t)$ convolution. In this study, we investigate and compare the performance of two TFD's in analyzing biological signals.

\section{The Wigner-Ville Distribution}

The Wigner-Ville distribution (WVD) of a continuous signal $z(t)$ is defined as [12]:

$$
W V(t, f)=\int_{-\infty}^{\infty} z\left(t+\frac{\tau}{2}\right) z^{*}\left(t-\frac{\tau}{2}\right) e^{-j 2 \pi f \tau} \mathrm{d} \tau
$$

Where, $f$ is the frequency variable. The WVD satisfies a large number of desirable mathematical properties. In particular, WVD is always real-valued; it preserves time and frequency shifts and satisfies the marginal properties, which yields the instantaneous power and the power spectrum. Based on (2), the WVD, which utilizes a time-only kernel $G(t, \tau)=G(t)=\delta(t)$ with $g(v, \tau)=g(v)=1$, has significant oscillatory cross-terms without a controlling factor, where the cross-terms can be larger in amplitude than the auto-terms. However, using a low-pass time-only kernel other than $\delta(t)$ will result in controlling the cross-terms by the low-pass function [12].

\section{The Bessel Distribution}

Bessel distribution (BD) for a real valued signal $f$ is defined as, [13]

$$
\begin{aligned}
B D_{f}(t, \omega) & =\int_{\tau} e^{-j \omega \tau} \int_{\mu} \frac{2}{\pi} \alpha|\tau| \sqrt{1-\left(\frac{\mu-t}{\alpha \tau}\right)^{2}} \\
& \times \Pi\left(\frac{\mu-t}{2 \alpha \tau}\right) f\left(\mu+\frac{\tau}{2}\right) f^{*}\left(\mu-\frac{\tau}{2}\right) \mathrm{d} \mu \mathrm{d} \tau
\end{aligned}
$$



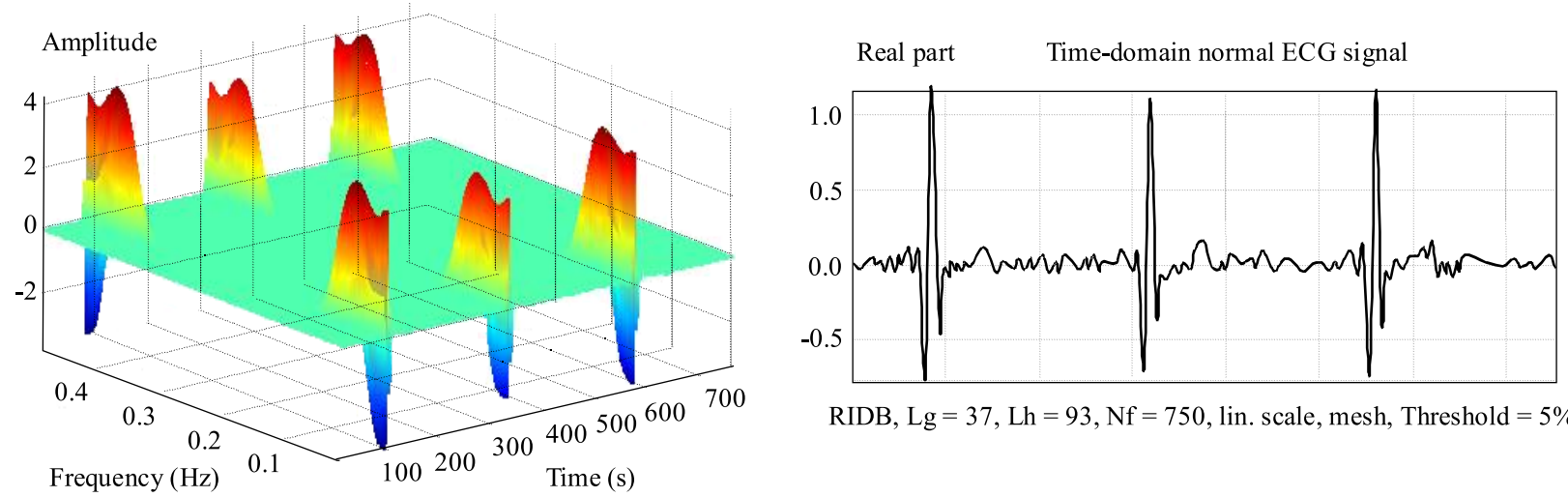

RIDB, $\mathrm{Lg}=37, \mathrm{Lh}=93, \mathrm{Nf}=750$, lin. scale, mesh, Threshold $=5 \%$

Fig. 1. Time and 3D time-frequency representations of the normal ECG signal using BD. The sampling frequency was $f_{s}=325$ samples/second
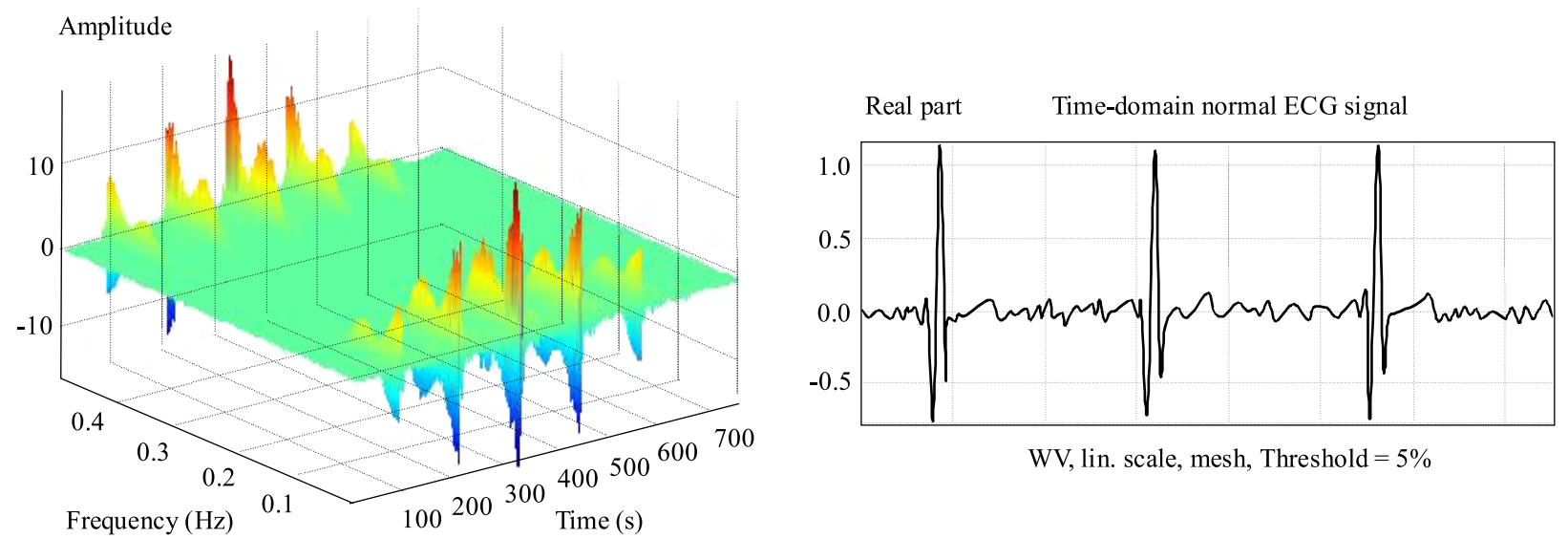

$\mathrm{WV}$, lin. scale, mesh, Threshold $=5 \%$

Fig. 2. Time and 3D time-frequency representations of the normal ECG signal using WVD. The sampling frequency was $f_{s}=325$ samples/second

Bessel distribution is a real valued distribution and it preserves the time and frequency-shifting properties, which holds similar behavior such as Fourier transforms application. It is also noticed that the time and frequency marginally associate with the instantaneous signal energy and the spectral energy density in the first order Bessel distribution. Also its first order moments correspond to the instantaneous frequency and group delay of the signal, and it preserves the time and frequency support properties with a recommended scaling factor $\alpha \leqslant 0.5$.

By using RID kernel design technique as described by Jeong and William in [9] we suggest a distribution based on Bessel function of the first kind. This distribution is defined as Reduced Interference Distribution based on Bessel function (RIDB);

$$
R I D B_{f}(t, v)=\int_{-\infty}^{+\infty} h(\tau) R_{f}(t, \tau) e^{-j 2 \pi v \tau} \mathrm{d} \tau
$$

with

$$
\begin{aligned}
R_{f}(t, \tau)=\int_{t-|\tau|}^{t+|\tau|} & \frac{2 g(\mu)}{\pi|\tau|} \sqrt{1-\left(\frac{\mu-t^{2}}{\tau}\right)^{2}} \\
& \times f\left(\mu+\frac{\tau}{2}\right) f *\left(\mu-\frac{\tau}{2}\right) \mathrm{d} \mu
\end{aligned}
$$

where $f$ is the original signal, $t$ is time instants, $\tau$ is time lag, $g$ is time smoothing window, and $h$ is frequency smoothing window.

\section{A COMPARISON OVER NORMAL AND ABNORMAL CARDIAC SIGNALS}

In this section, we compare the performance of the Bessel distribution with WVD over normal and abnormal cardiac signals. Equation (5) is used to find out the results of Bessel distribution for cardiac signals. 

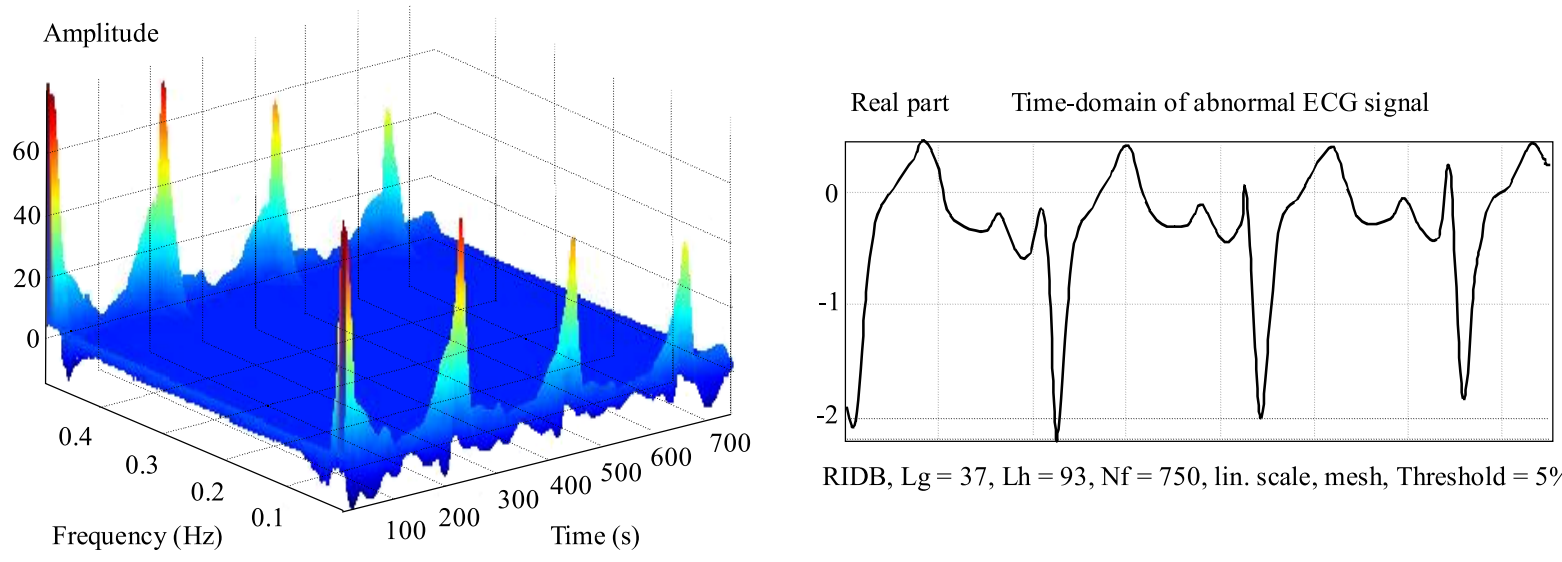

RIDB, $\mathrm{Lg}=37, \mathrm{Lh}=93, \mathrm{Nf}=750$, lin. scale, mesh, Threshold $=5 \%$

Fig. 3. . Time and 3D time-frequency representations of the abnormal ECG signal (Heart Failure) using BD. The sampling frequency was $f_{s}=325$ samples/second
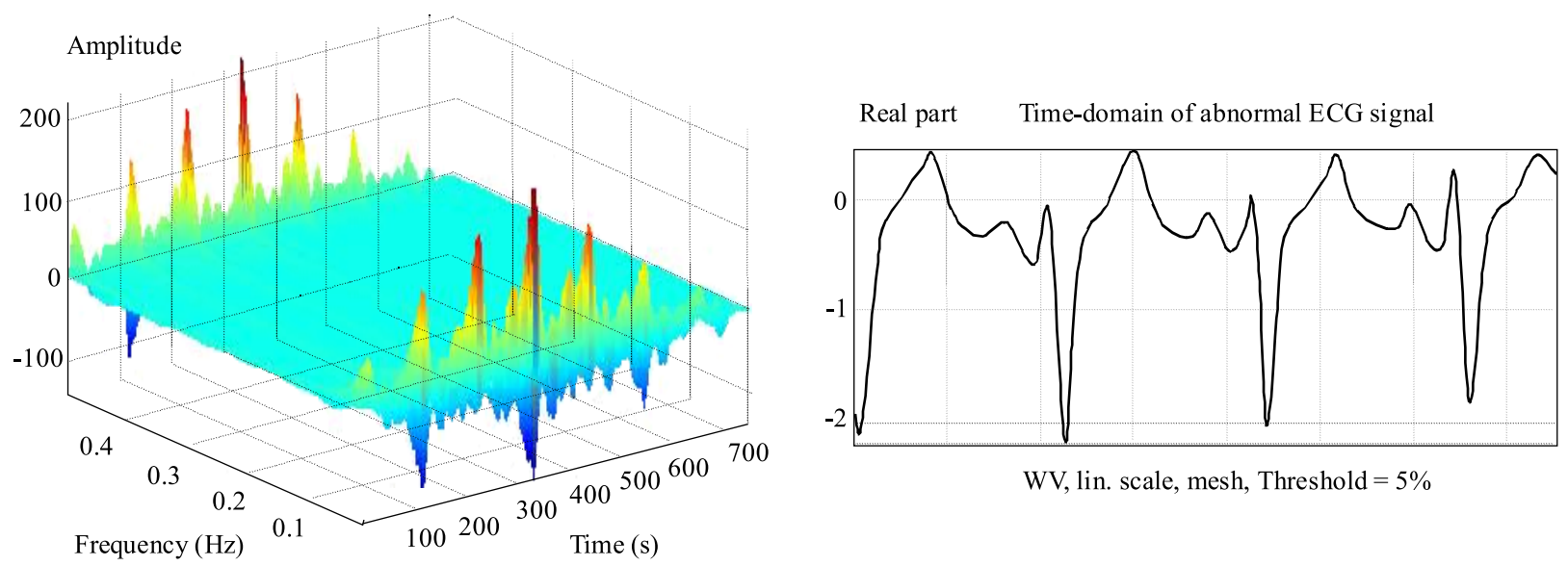

Fig. 4. Time and 3D time-frequency representations of the abnormal ECG signal (Heart Failure) using WVD. The sampling frequency was $f_{s}=325$ samples/second

\subsection{Comparison over Normal ECG Signal}

A normal ECG signal of length 3 seconds was converted by Hilbert transform into its analytical forms and processed using the BD and the WVD. Figure 1 and Fig. 2 show the time and 3D time-frequency representations of this signal, normal ECG, utilizing BD and WVD, respectively. Also from these figures, one observes that the BD has best resolution, as it can reveal the frequency components of the normal ECG signal with a resolution much higher than that given by WVD. The cross-terms in WVD make the task of identifying the QRS peaks and T-wave extremely difficult.

\subsection{Comparison over Abnormal ECG}

A length of 3 seconds of the heart failure signal is processed using the proposed distribution, and the WVD. This signal has normal QRS complexes duration of 0.1second width and a short P-R interval. The QRS com- plexes have irregular shapes. Fig. (3) and Fig. (4) show the time and 3D time-frequency representations of this signal utilizing BD and WVD, respectively. The proposed distribution manages to detect the three QRS complexes in the signal with their exact shape; however, the WVD fails to track changes in the frequency components of the QRS complex of this signal due to cross terms. All frequency components in this signal are clearly revealed by the BD.

\section{CONCLUSIONS}

The BD could be considered as a member of the RIDs when $\alpha \leqslant 0.5$ where the distribution satisfies the finite support properties. The heart signal is essentially a nonstationary transient deterministic signal generated by the closure and vibration of the heart valve in a dynamic system which generates other acoustic events. The presented TFDs are able to detect the QRS complexes in these sig- 
nals; however, WVD fail to track changes (abnormality) in the frequency components of the QRS complexes of these signals. The BD has revealed the true structure of the QRS complexes in these signals where there are two components with narrow separation in frequency. As such a high-resolution time frequency distribution can be a pre-process towards automatic time-frequency heart failure detection and classification.

\section{REFERENCES}

[1] BIGAN, C.-WOOLFSON, M. S.: Time-frequency analysis of short segments of biomedical data, IEEE Proc.-Sci. Meas. Technol. 147 No. 6 (November 2000), 368-373.

[2] CLAYTON, R. H.-MURRAY, A. : Estimation of the ECG signal spectrum during ventricular fibrillation using the fast Fourier transform and maximum entropy methods, Proceedings of the Computers in Cardiology, September 1993, pp. 867-870.

[3] WOOD, J. C.-BARRY, D. T.: Time-frequency analysis of skeletal muscle and cardiac vibrations, Proceedings of the IEE 84 No. 9 (September 1996), 1281-1294.

[4] COHEN, L.: Time-frequency distributions-a review, Proc. IEEE 77 No. 7 (1989), 941-981.

[5] COHEN, L.: Time-Frequency Analysis, Prentice Hall PTR, Englewood Cliffs, New Jersey, 1995.

[6] HUSSAIN, Z. M.-BOASHASH, B.: Adaptive instantaneous frequency estimation of multi-component FM signals using quadratic time-frequency distributions, IEEE Trans. Signal Process. 50 No. 8 (2002), 1866-1876.

[7] FLANDRIN, P.: Time-Frequency/Time-Scale Analysis, Academic Press, Boston, MA, 1998.

[8] CHOI, H.-WILLIAMS, W.: Improved time-frequency representation of multicomponent signals using exponential kernels, IEEE Trans. Signal Process. 37 No. 6 (1989), 862-871.
[9] JEONG, J.-WILLIAMS, W.: Kernel design for reduced interference distributions, IEEE Trans. Signal Processing $\mathbf{4 0}$ No. 2 (1992), 402-412.

10] Physiobank, Physionet, Physiologic signal archives for biomedical research, http://www.physionet.org/physiobank/, viewed August 2005.

11] HUSSAin, Z. M.-BOASHASH, B.: Design of time-frequency distributions for amplitude and IF estimation of multicomponent signals, International Symposium on Signal Processing and Its Applications (ISSPA'2001), vol. 1, August 2001, pp. 339-342.

[12] STANKOVIC, L. J.-KATKOVNIC, V.: The Wigner distribution of noisy signals with adaptive time-frequency varying window, IEEE Trans. Signal Process 47 No. 4 (1996), 1099-1108.

[13] GUO, Z.-DURAND, L.-G.-HOWARD, C. L. : The Time-Frequency Distributions of Nonstationary Signals Based on a Bessel Kernel, IEEE Trans. Signal Process. 42 No. 7 (1994), 1700-1707.

Received 11 November 2010

Allam Mousa received his BSc, MSc and PhD in Electrical and Electronics Engineering from Eastern Mediterranean University in 1990, 1992 and 1996 respectively. He is an Associate Professor at the Department of Electrical Engineering An Najah University. During 2002-2009 he was the chairman of the same department and was the chairman of the Electronics Engineering Department Al Quds University in 19992000. His current research interests are in the field DSP include speech and image processing and Telecommunications. He is also SMIEEE and member of Engineering Association.

Rashid Saleem received his BS in Electronic Engineering in 1999 from Ghulam Ishaq Khan Institute of Engineering Sciences and Technology and MS in Computer Engineering from University of Engineering and Technology Taxila Pakistan in 2006. Currently he is pursuing his PhD in Electrical and Electronic Engineering at the University of Manchester, UK and his recent research interest is in the field of Ultra Wideband system modeling and performance evaluation.

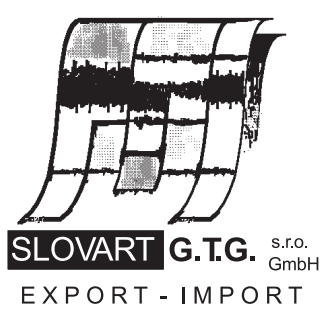

EXPORT - IMPORT
EXPORT - I M P OR T

of periodicals and of non-periodically printed matters, books and CD-ROMs

Krupinská 4 PO BOX 152, 85299 Bratislava 5, Slovakia tel: ++421263839 472-3, fax: ++421263839485 info@slovart-gtg.sk; http://www.slovart-gtg.sk

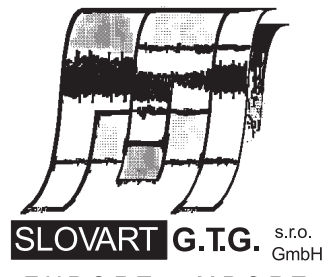

EXPORT - IMPORT 